\title{
EXTENSION OF POSITIVE HOLOMORPHIC LINE BUNDLES
}

\author{
BY BERNARD SHIFFMAN ${ }^{1}$
}

Communicated by Gian-Carlo Rota, May 7, 1971

In this note, we announce a result on extending complex line bundles through subvarieties of codimension 2. The motivation for this result is that it allows us to extend a recent result of Phillip Griffiths [2] on meromorphically extending holomorphic maps into compact Kahler manifolds. Details and further related results will appear elsewhere.

A holomorphic line bundle $L$ on a complex manifold $M$ is said to be semipositive if there exists a hermitian metric $h$ on $L$ such that the curvature form

$$
\Omega=\frac{i}{2 \pi} \partial \bar{\partial} \log h
$$

is positive semidefinite at all points of $M$ (i.e., the locally defined functions $\log h$ are plurisubharmonic).

Theorem. Let $M$ be a complex manifold, and let $S$ be an analytic set in $M$ such that codim $S=2$. Then every semipositive holomorphic line bundle $L$ on $M-S$ extends to a holomorphic line bundle on $M$.

If codim $S \geqq 3$, then it is a well-known fact that any line bundle $L$ on $M-S$ extends to $M$ (see [3]).

In order to prove the theorem, one must show that $L$ induces the zero element of $H_{S}^{2}\left(M, \mathcal{O}^{*}\right) \approx \Gamma\left(M, \mathfrak{F}_{S}^{2} \mathcal{O}^{*}\right)$. Therefore it suffices to show that $L$ extends locally, and the theorem is then a consequence of the following lemma applied to the curvature form $\Omega$.

We let $D$ denote the open unit disk in $\boldsymbol{C}$.

Lemma. Let

$$
\omega=i \sum f_{\alpha \beta} d z_{\alpha} \wedge d \bar{z}_{\beta} \quad(1 \leqq \alpha, \beta \leqq n)
$$

AMS 1970 subject classifications. Primary 32D15, 53C55; Secondary 32C10, $32 \mathrm{H} 99$.

Key words and phrases. Holomorphic line bundle, curvature form, positive line bundle, sheaf cohomology, local cohomology, harmonic function, plurisubharmonic function, analytic subvariety, Kahler manifold, meromorphic map.

1 This research was partially supported by National Science Foundation Grant GP-21193. 
be a real closed $(1,1)$-form on the domain

$$
W=\left(D^{2}-0\right) \times D^{n-2} \subset C^{n} .
$$

If $f_{11} \geqq 0$ and $f_{22} \geqq 0$ on $W$, then there exists a real-valued function $u$ on $W$ such that $\omega=d d^{c} u$. In particular, if $\omega$ is a Kahler form on $W$, then $\omega=d d^{c} u$, where $u$ is a function on $W$.

Write $W=W_{1} \cup W_{2}$, where

$$
W_{j}=\left\{z \in W: z_{j} \neq 0\right\}, \text { for } j=1,2 .
$$

Then $\omega \mid W_{j}=d d^{c} u_{j}$, where $u_{j}$ is a real-valued function on $W_{j}(j=1,2)$. Let $h=u_{1}-u_{2}$ on $W_{1} \cap W_{2}$. Then $d d^{c} h=0$, i.e., $h$ is pluriharmonic. For the case $n=2, u_{1}$ and $u_{2}$ are subharmonic in each variable separately. The main point of the lemma (and the theorem) is that we can then write $h=h_{1}-h_{2}$, where $h_{j}$ is a pluriharmonic function on $W_{j}(j=1,2)$, and therefore $u=u_{j}-h_{j}$ is a globally defined function on $W$ with $\omega=d d^{c} u$. The proof uses the solution of the Dirichlet problem on the annulus $A_{r}=\{r<|z|<1\}$. By considering the biannulus $A_{r} \times A_{s}$ and letting $(r, s) \rightarrow(0,0)$, one constructs functions $\tilde{u}_{j}$ on $W_{j}(j=1,2)$ such that $\tilde{u}_{1}$ and $\tilde{u}_{2}$ are harmonic in each variable separately, and $h=\tilde{u}_{1}-\tilde{u}_{2}$. The existence of $h_{1}$ and $h_{2}$ then follows from the equation $h=\tilde{u}_{1}-\tilde{u}_{2}$.

In [2], Phillip Griffiths proved the following result.

Theorem (GRIfFIths). Let $f: D^{n}-0 \rightarrow X$ be a holomorphic map, where $X$ is a compact Kahler manifold. If $n \geqq 3$, then $f$ extends meromorphically to $D^{n}$ (i.e., the closure of the graph of $f$ is an analytic set in $\left.D^{n} \times X\right)$.

Griffiths' idea is to apply a theorem of Errett Bishop [1], [4] on extending analytic sets with finite volume. Consider the Kahler form

$$
\omega=\frac{i}{2} \sum d z_{\alpha} \wedge d \bar{z}_{\alpha}+f^{*} \omega_{X}
$$

on $D^{n}-0$, where $\omega_{X}$ is the given Kahler form on $X$. The volume of the graph of $f$ is then given by $\int \omega^{n}$. Since $H^{2}\left(D^{n}-0, R\right)=0$ and $H^{1}\left(D^{n}-0, \mathcal{\theta}\right)=0$, for $n \geqq 3$, one can write $\omega=d d^{c} u$, where $u$ is a plurisubharmonic function on $D^{n}-0$. By approximating $u$ by smooth plurisubharmonic functions on a ball $B \subset \subset D^{n}$ about 0 and by applying Stokes' theorem, Griffiths concludes that $\int_{B}\left(d d^{c} u\right)^{n}<+\infty$.

By the above lemma, we can also write $\omega=d d^{c} u$ for the case $n=2$ (although $H^{1}\left(D^{2}-0, \mathcal{\theta}\right) \neq 0$ ). Therefore, Griffiths' theorem is also valid for $n=2$. 


\section{REFERENCES}

1. E. Bishop, Conditions for the analyticity of certain sets, Michigan Math. J. 11 (1964), 289-304. MR 29 \#6057.

2. P. A. Griffiths, Two theorems on extensions of holomorphic mappings, Invent. Math. (to appear).

3. G. Scheja, Riemannsche Hebbarkeitssätze für Cohomologieklassen, Math. Ann. 144 (1961), 345-360. MR $26 \# 6437$.

4. B. Shiffman, Extending analytic subvarieties, Proc. Sympos. on Several Complex Variables (Park City, Utah, 1970), Lecture Notes in Math., no. 184, Springer-Verlag, Berlin, 1971, pp. 208-222.

Yale University, New Haven, Connecticut 06520 